Research Paper

\title{
Postmortem evaluation of brain edema: An attempt with measurements of water content and brain-weight-to-inner-skull-circumference ratio
}

Johanna Marie Lundesgaard Eidahl ${ }^{\mathrm{a}, \mathrm{b}, ~}$ *

joheid@ous-hf.no

Siri Hauge Opdal ${ }^{\mathrm{a}}$

siropd@ous-hf.no

Torleiv Ole Rognum ${ }^{\mathrm{a}, \mathrm{b}}$

trognum@ous.hf.no, t.o.rognum@medisin.uio.no

Arne Stray-Pedersen ${ }^{\mathrm{a}, \mathrm{b}}$

arnest@ous-hf.no, arne.stray-pedersen@medisin.uio.no

${ }^{a}$ Department of Forensic Sciences, Group of Paediatric Forensic Medicine, Oslo University Hospital, Oslo, Norway

${ }^{\mathrm{b}}$ Institute of Clinical Medicine, University of Oslo, Oslo, Norway

*Corresponding author. Avdeling for rettsmedisinske fag, OUS HF, Seksjon for Rettsmedisinske undersøkelser av barn, Postboks 4950 Nydalen 0424, Oslo, Norway. 


\begin{abstract}
Introduction

Postmortem evaluations of cerebral edema typically involve examinations of macroscopic features such as the presence of pressure signs and compression of the ventricles. Global massive edema is easily detectable in an autopsy, but less-extensive edema may be difficult to diagnose.
\end{abstract}

\begin{abstract}
Aim
The aim of this study was to compare measurements of brain water contents, postmortem CT radiodensity and brain weight to skull size in edematous and nonedematous brains in order to develop an objective method for postmortem evaluations of brain edema.
\end{abstract}

\title{
Method
}

Fifty-four subjects autopsied at Oslo University Hospital underwent a standard forensic postmortem examination, including a computed axial tomography (CT) scan, measurement of brain weight, and macroscopic evaluation of the brain. CT images were used to roughly measure the inner skull circumference. The water content of the brain was determined by excising samples of approximately $1 \mathrm{~g}$ of brain tissue from eight different areas of the brain surface, drying them, and measuring their percentage water content.

\section{Results}

The main finding was a significant relationship between brain weight and inner skull circumference, with the ratio between these two parameters being significantly higher in cases with severe postmortem brain edema than in cases with very little or no brain edema. The water content did not differ significantly between the edema and nonedema cases. There were no significant changes in radiodensity. 


\section{Conclusion}

This indicates that the brain-weight-to-inner-skull-circumference ratio may serve as a good marker for severe brain edema in postmortem diagnostics, whereas measurements of water content can be misleading.

Keywords: Cerebral edema; Water content; Skull circumference; Brain weight; Brain-weight-toinner-skull-circumference ratio. 


\section{Introduction}

Cerebral edema results from the accumulation of fluid in the brain parenchyma, which causes a volumetric enlargement of the brain and is a known complication of numerous pathological conditions and injuries. Severe brain edema may result in irreversible and fatal injuries. The two most common types of cerebral edema encountered are vasogenic and cytotoxic (1). Vasogenic edema is mainly extracellular fluid accumulation caused by a disrupted blood-brain barrier due to e.g. trauma, infarction or infection. Cytotoxic edema is characterized by intracellular swelling and is most commonly due to hypoxia and ischemic conditions. Cytotoxic edema occurs when cells fail to maintain the ATP-dependent sodium/potassium membrane pumps, resulting in sodium accumulating within cells and a net influx of water into cells, and may evolve even in the presence of an intact blood-brain barrier (1).

In clinical practice, brain edema is most commonly assessed by neuroimaging with computed axial tomography $(\mathrm{CT})$ or magnetic resonance imaging and measurement of intracranial pressure (2). In fatalities, global massive edema is easily detectable upon autopsy by examination with the naked eye, but less-extensive edema may be difficult to establish. A postmortem diagnosis of brain edema traditionally includes measurement of the brain weight and an evaluation of macroscopic features such as gyral flattening and compression of the sulci, as well as looking for asymmetry and impression marks on the basal parts of the brain, such as grooving of the temporal unci and extension of the cerebellar cone (3). An abnormal brain weight of more than $1500 \mathrm{~g}$ is also used as a sign of edema (4), but a heavy brain may be the result of simple brain swelling due to blood congestion in the terminal phase (3). In our experience the diagnosis of edema will frequently differ between the neuropathologist examining the fixed brain and the forensic pathologist performing the autopsy. Formaldehyde fixation alters the texture of the brain and may result in changes on the surface to the gyri, grooves, and sulci appearing more prominent. Sectioning of the brain makes it possible to examine the ventricles and evaluate potential compression due to edema. Histopathological examinations can reveal secondary hemorrhage that may result from herniation, but this depends on the severity and duration of the edema before death occurred. Also, pale hematoxylin-and-eosin 
staining, broadening of perivascular spaces, and a generally sponge-like appearance of the tissue may be indicators of edema. Congestion of intracerebral vessels with perivascular hemorrhages is a feature more typical of congestive brain swelling. Yet, discriminating edema from brain swelling and postmortem changes may be challenging (3).

Several attempts have been made to develop a more objective method for postmortem evaluations of brain edema. Röthig and Schaarschmidt presented a formula in 1977 in which the theoretical brain weight was a linear function of body height (5). They considered that a brain weighing more than the mean plus one standard deviation of the theoretical normal brain weight for a certain body height should be considered edematous. Madro and Chagowski suggested that specific weight measurements of various parts of the brain provides a better indicator of brain edema than conventional macroscopic and microscopic evaluation (6). Hausmann et al. used the formula of Röthig and Schaarschmidt to verify brain edema, and found no statistical correlation between macroscopic pressure signs and increased brain weight (7). They also found no significant correlation between increased brain weight and perivascular distension observed in histology slides (7). Radojevic et al. very recently argued that not only the weight of the brain but also its volume plus that of the cranial cavity must be taken into account when evaluating potential brain edema (8). Berger et al. had some success in diagnosing edema when using postmortem CT images to assess narrowing of the temporal horns and herniation of the cerebellar tonsils (9). Objective measurements of the radiodensities of the gray matter and white matter did however not seem able to discriminate between edema and nonedema (9). White matter attenuation seems to increase post mortem, which is believed to be due to agonal brain swelling (10). The significance of CT findings is not yet clarified as the literature on this area is limited.

In the present study we measured water content in brain tissue sampled at autopsy, hypothesizing that brains with edema have increased water content compared to nonedema cases. Furthermore, we hypothesized that brain edema causes changes in the radiodensity measured in CT images of the brain, and thus a change in the ratio between the gray matter attenuation and the white matter attenuation. Finally we hypothesized that edema cases have larger brain weights compared to 
the inner skull size than non-edema cases and investigated the ratio between the brain weight and the approximated inner skull circumference based on CT imaging.

\section{Materials and methods}

\subsection{Subjects}

This study included 54 subjects (33 males and 21 females) with a median age at death of 44.2 years (age range $=4-89$ years). All subjects underwent a standard forensic postmortem examination, which included whole-body CT scan in addition to a forensic autopsy. The cases were collected consecutively during 2015-2016, and the autopsies were performed at the Department of Forensic Sciences, Oslo University Hospital (formerly called the Norwegian Institute of Public Health). All subjects were autopsied within 5 days postmortem (median $=2$ days, range $=0-5$ days), with only one case having a postmortem time exceeding 3 days. The characteristics of the subjects are given in Table 1.

Table 1: Case characteristics for all subjects included in this study.

\begin{tabular}{|c|c|c|c|c|c|c|c|}
\hline & $\begin{array}{l}\text { Cause of } \\
\text { death }\end{array}$ & $\begin{array}{l}\text { Number } \\
\text { of cases }\end{array}$ & $\begin{array}{l}\text { Sex, } \\
\text { males/females }\end{array}$ & $\begin{array}{l}\text { Age, } \\
\text { years }^{*}\end{array}$ & $\begin{array}{l}\text { Height, } \\
\text { cm }^{*}\end{array}$ & $\begin{array}{l}\text { Body } \\
\text { weight, } \text { kg }^{*}\end{array}$ & BMI, $\mathrm{kg} / \mathrm{m}^{2^{*}}$ \\
\hline \multirow[t]{4}{*}{$\begin{array}{l}\text { Edema cases } \\
\mathrm{N}=16\end{array}$} & Violence & 6 & $5 / 1$ & $\begin{array}{l}43 \\
(4-63)\end{array}$ & $\begin{array}{l}180 \\
(112-168)\end{array}$ & $\begin{array}{l}184 \\
(17-102)\end{array}$ & $\begin{array}{l}26.5 \\
(13.6-30.8)\end{array}$ \\
\hline & Asphyxia & 4 & $2 / 2$ & $\begin{array}{l}39 \\
(33-59)\end{array}$ & $\begin{array}{l}173 \\
(152-177)\end{array}$ & $\begin{array}{l}69 \\
(55-92)\end{array}$ & $\begin{array}{l}25.7 \\
(18.6-29.2)\end{array}$ \\
\hline & Intoxication & 5 & $2 / 3$ & $\begin{array}{l}34 \\
(18-52)\end{array}$ & $\begin{array}{l}172 \\
(159-188)\end{array}$ & $\begin{array}{l}97 \\
(60-111)\end{array}$ & $\begin{array}{l}32.8 \\
(23.7-34.5)\end{array}$ \\
\hline & Natural & 1 & $1 / 0$ & 36 & 179 & 90 & 28.1 \\
\hline \multirow{2}{*}{$\begin{array}{l}\text { Controls } \\
\text { (non- } \\
\text { edematous) }\end{array}$} & Violence & 5 & $4 / 1$ & $\begin{array}{l}64 \\
(29-70)\end{array}$ & $\begin{array}{l}174 \\
(159-183)\end{array}$ & $\begin{array}{l}76 \\
(44-103)\end{array}$ & $\begin{array}{l}27.5 \\
(17.4-31.8)\end{array}$ \\
\hline & Asphyxia & 6 & $2 / 4$ & 50 & $173(151-$ & 59 & 22.0 \\
\hline
\end{tabular}




\begin{tabular}{|c|c|c|c|c|c|c|c|}
\hline \multirow[t]{3}{*}{$\mathrm{N}=38$} & & & & $(25-64)$ & 185) & $\begin{array}{l}(51-97) \\
\end{array}$ & $(14.9-32.0)$ \\
\hline & Intoxication & 12 & $9 / 3$ & $\begin{array}{l}37 \\
(22-64)\end{array}$ & $\begin{array}{l}174 \\
(168-192)\end{array}$ & $\begin{array}{l}84 \\
(52-109)\end{array}$ & $\begin{array}{l}28.0 \\
(17.6-31.5)\end{array}$ \\
\hline & Natural & 15 & $8 / 7$ & $\begin{array}{l}56 \\
(29-89)\end{array}$ & $\begin{array}{l}171 \\
(159-185)\end{array}$ & $\begin{array}{l}73 \\
(49-108)\end{array}$ & $\begin{array}{l}25.7 \\
(17.4-36.5)\end{array}$ \\
\hline Total & & 54 & $33 / 21$ & $\begin{array}{l}44 \\
(4-89)\end{array}$ & $\begin{array}{l}172 \\
(112-192)\end{array}$ & $\begin{array}{l}80 \\
(17-111)\end{array}$ & $\begin{array}{l}26.8 \\
(13.6-36.5)\end{array}$ \\
\hline
\end{tabular}

* median (range) values

Age, sex, height, body weight, cause of death, and other necessary information were collected from the forensic autopsy reports. The only child included in our study group was 4 years of age; the second youngest subject was 17 years. Each case was categorized into one of the following four categories after reviewing the circumstances and the cause of death:

1. Violent deaths were fatalities due to any kind of fatal trauma, such as accidents, iatrogenic injuries, and homicides. Three cases had sustained severe blunt head trauma.

2. Asphyxia comprised deaths by hanging, drowning, or other mechanical breathing obstruction.

3. Intoxication comprised deaths due to drug poisoning and also hypothermia (due to the similarities in death mechanisms).

4. Natural deaths were sudden unexpected deaths due to pathological conditions, which were mainly of cardiovascular origin.

\subsection{Macroscopic evaluation}

During the autopsy the whole brain was removed from the skull and immediately examined for signs of brain edema. The degree of brain edema was rated on a scale from 0 to 3 , with 0 being no brain edema and 3 being severe brain edema. The evaluation comprised an inspection of the traditional macroscopic signs of brain edema; gyral flattening of the brain surface (score of 1), any compression of the ventricles (score of 2), or impression marks on the base of the brain (temporal unci and cerebellar cone) (score of 3). The gross evaluation of the ventricles was performed using postmortem 
CT images. The cases were divided in two groups: those with a score of 2 or 3 were considered to have brain edema, while those with a score of 0 or 1 were considered to be nonedematous. Formalin fixation was applied to edema cases for neuropathological examinations and verification. All other cases considered nonedematous by the examining physician were dissected fresh during the autopsy (coronal sectioning of the cerebrum, midline splitting and coronal sectioning of the cerebellum and horizontal/axial sectioning of the brain stem). Only cases with no pathological findings were included in the nonedema group.

\subsection{Inner skull circumference}

The inner sagittal and biparietal distances of the skull were determined on CT images of the head by two of the authors (JMLE and ASP). After orienting the images in three axes, the maximum anteroposterior distance (longitudinal distance) from the interior of the frontal bone to the occipital bone was measured (Fig. 1a). Wrinkles and bumps in the frontal and occipital bones were avoided. The axial plane was somewhat above the frontal sinuses and the internal occipital protuberance (Fig. 1c). The maximum coronal inner skull distance (transverse distance) was measured on the same image view (Fig. 1b).

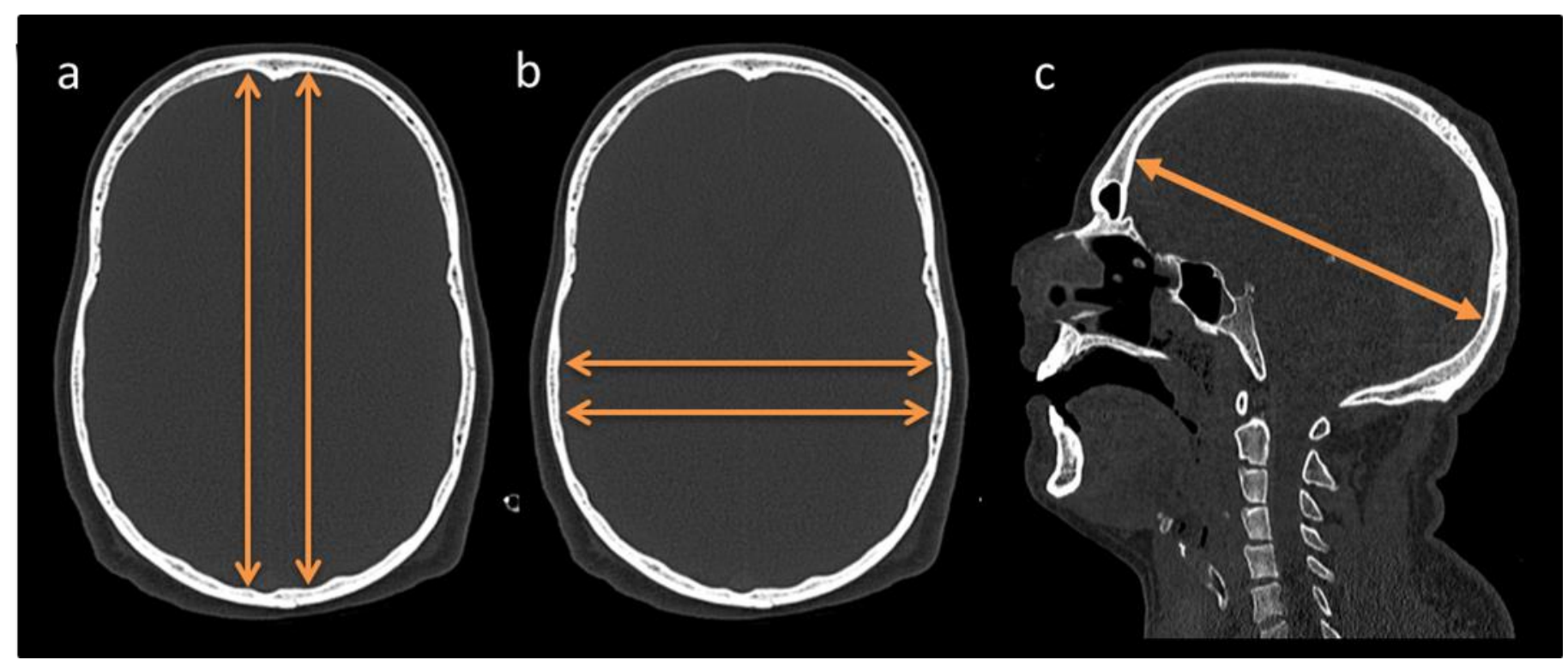

Fig. 1 Illustration of the measurements made on CT images. (a) The longitudinal distance was obtained by the average of two measurements performed either side of the sagittal midline. (b) The 
maximum transverse distance was measured on the same CT image, biparietal to the longitudinal measurements. (c) The axial plane was somewhat above the frontal sinuses and the internal occipital protuberance.

The inner skull circumference was approximated by dividing the measured distance into the radius and using the following formula for the circumference of an ellipse: $2 \pi \sqrt{ }\left(\left(a^{2}+b^{2}\right) / 2\right)$. The ratio between the brain weight measured at autopsy and the inner circumference of the skull was calculated.

\subsection{Measurement of radiodensity}

Image acquisition was performed on a dual-source CT scanner (SOMATOM Flash Definition, Siemens, Germany). The scans were performed shortly prior to autopsy at $120 \mathrm{kV}$ and with automatic dose modulation. A separate head scan (slice thickness $=0.6 \mathrm{~mm}$, increment $=0.4 \mathrm{~mm}$, and reference current=600 mAs) was performed. Two regions of interest (ROIs) were defined in the CT images for each case (Fig. 2). Each ROI was a circle with an area of $25-30 \mathrm{~mm}^{2}$ : one in the gray matter in the frontal region, and one in the white matter in the inner part of the parietal region of the brain. The radiodensity was measured in each ROI and expressed in Hounsfield units (HU). The gray-matter-towhite-matter radiodensity ratio was calculated for each individual, in accordance with Berger et al. (9).

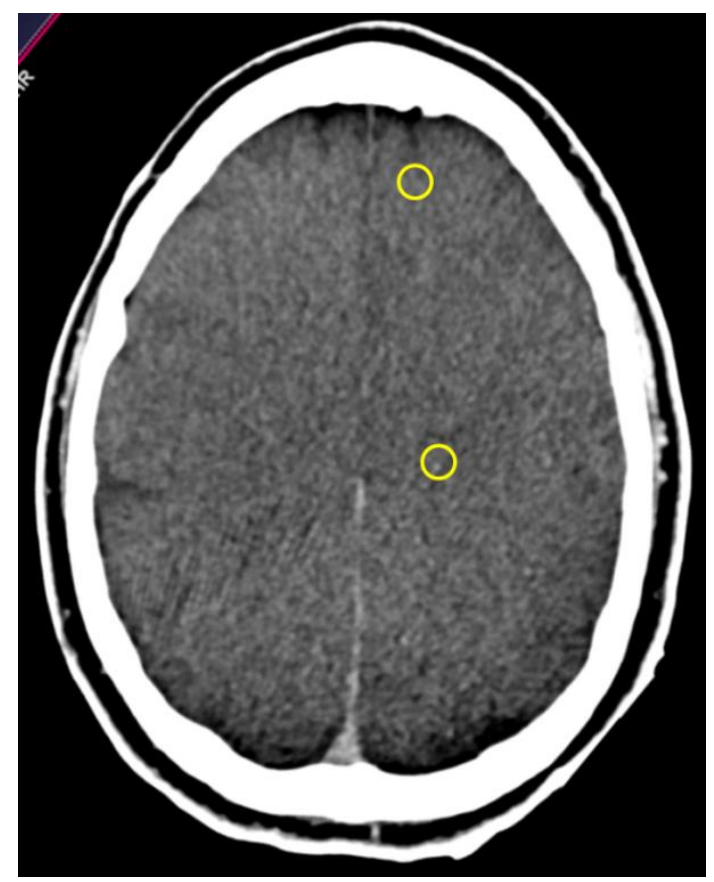

Fig. 2 Regions of interest in gray matter and white matter for radiodensity measurements. 


\subsection{Determination of water content}

Small samples of brain tissue weighing approximately $1 \mathrm{~g}$ were excised from the temporal, frontal, and occipital regions of both cerebral hemispheres, as well as from the posterior part of the cerebellum bilaterally. The samples consisting of gray matter and white matter were taken within a few minutes after removing the brain from the skull, immediately after performing the gross examination. After determining the wet weight of the samples in grams using precision scales to four decimal places, the samples were placed in small containers and stored at $-76^{\circ} \mathrm{C}$.

The water content of the samples was determined by drying them for 24 hours at $95^{\circ} \mathrm{C}$ in an oven attached to a vacuum pump. The remaining solid material weight was then assessed for each sample. The result was expressed as the brain water content as a percentage: (wet weight - dry weight $) /($ wet weight $) \times 100$.

\subsection{Statistical analysis}

Scores, ratios, and percentages of water content were compared between the following three groups using the nonparametric Kruskal-Wallis test: edema, nonedema, and late edema (the latter group explained below). The brain weight and brain-weight-to-inner-skull-circumference ratio was compared between the edema and nonedema groups using a Mann-Whitney $U$ test. In order to evaluate the intra- and interindividual reproducibility of the skull CT-measurements by the two observers a Cronbach's alpha test was performed.

Regression analysis was performed to find the closest-fit lines for the edema and nonedema groups in scatter plots. This also yielded equations for the two regression lines, their confidence curves, and their confidence intervals.

All statistical analyses were performed using IBM SPSS Statistics, version 23.0 (SPSS, Chicago, IL, USA). 


\section{Results}

The gross macroscopic examination resulted in 16 of the 54 brains examined being categorized as edematous. The presence of edema was confirmed in all cases by neuroimaging in the terminal phase (12/16), neuropathological examination $(15 / 16)$, or both. The remaining 38 cases were regarded as nonedematous.

The causes of death in the edema cases were violence $(n=6)$, intoxication $(n=5)$, asphyxia $(\mathrm{n}=4)$, and natural $(\mathrm{n}=1)$. Thirteen of these 16 cases $(81.25 \%)$ had experienced a sudden respiratory and/or circulatory collapse, and after resuscitation had been subject to intensive-care treatment for more than 8 hours before death finally occurred. In four of the edema cases that were resuscitated after the initial collapse (one each of asphyxia, intoxication, natural, and violence), life support was applied for more than 5 days. In all of these cases the brain edema were accompanied by signs of severe tissue damage and early atrophy, and so these cases were placed in a separate category (the late edema group, $\mathrm{n}=4)$. This left the edema-group with 12 cases.

The results obtained in the measurements of brain weight, estimation of inner skull circumference, calculation of the brain-weight-to-inner-skull-circumference ratio, and average water content measurements are summarized in Table 2. 
Table 2: Measured brain weight, estimated inner skull circumference, brain-weight-to-inner-skullcircumference ratio, average water content measurements, and gray-matter-to-white-matter radiodensity ratio.

\begin{tabular}{|l|l|l|l|l|}
\hline Parameter & Edema $(\mathbf{n = 1 2})$ & Nonedema & Late edema $(\mathbf{n = 4})$ & $\mathbf{p}^{*}$ \\
\hline Brain weight, g & $1535(1450-$ & $1387.5(1180-$ & $1420(1240-1510)$ & $<\mathbf{0 . 0 0 1}$ \\
& $1995)$ & $1700)$ & & \\
\hline Inner skull circumference, cm & $46.3(43.5-51.9)$ & $46.2(43.7-52.0)$ & $45.3(43.7-49.0)$ & 0.667 \\
\hline $\begin{array}{l}\text { Brain-weight-to-inner-skull- } \\
\text { circumference ratio }\end{array}$ & $33.6(31.3-38.5)$ & $29.6(26.3-33.8)$ & $30.7(28.4-31.7)$ & $<\mathbf{0 . 0 0 1}$ \\
\hline $\begin{array}{l}\text { Average percentage water } \\
\text { content in the entire brain }\end{array}$ & $81.8(79.7-84.3)$ & $81.37(80.1-84.2)$ & $81.8(80.5-84.5)$ & 0.733 \\
\hline $\begin{array}{l}\text { Gray-matter-to-white-matter } \\
\text { radiodensity ratio }\end{array}$ & $1.14(1.00-1.66)$ & $1.11(1.00-1.42)$ & $1.05(1.02-1.14)$ & 0.322 \\
\hline
\end{tabular}

Data are median (range) values. *Kruskal-Wallis test

Within the three groups significant differences in the brain weight $(\mathrm{p}<0.001)$ and the ratio between brain weight and the inner skull circumference $(\mathrm{p}<0.001)$ were observed. The edema cases had significantly higher brain weight than the nonedema cases $(\mathrm{p}<0.001)$. There was no significant intergroup difference in the gray-matter-to-white-matter radiodensity ratio. Intra- and interindividual reproducibility of the skull measurements in CT-images showed good agreement ( $\alpha=0.859$ and $\alpha=0.938$ respectively).

The measured brain weight was plotted against calculated skull circumference, and a regression line was calculated for cases of edema and nonedema (Fig. 3). The regression equation given by the nonedema cases, the blue line in Fig. 3, may be used to calculate a theoretical normal brain weight. The regression equation given by the edema cases can be transcribed as follows:

$$
y=65.35 \times 2 \pi \sqrt{ }\left(\left((\text { longitudinal distance })^{2}+(\text { transverse distance })^{2}\right) / 2\right)-1460
$$




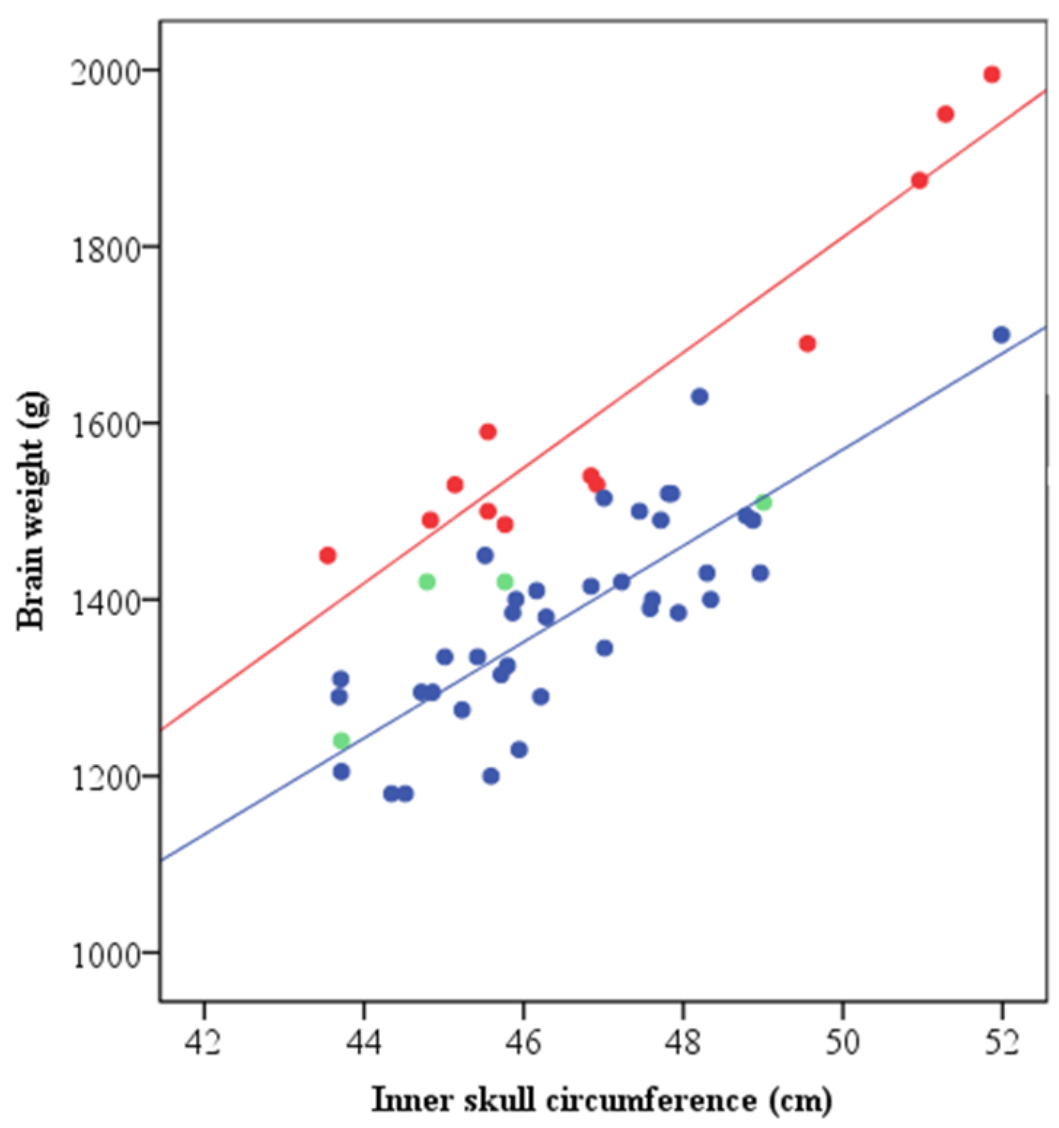

Fig. 3: Measured brain weight relative to the calculated inner skull circumference. The red dots and regression line $(y=65.35 x-1460)$ represent the edema cases $\left(\mathrm{R}^{2}=0.907\right)$, and the blue dots and regression line $(\mathrm{y}=54.51 \mathrm{x}-1160)$ represent the nonedema cases $\left(\mathrm{R}^{2}=0.666\right)$. The late edema cases are indicated separately by green dots; these cases had a brain-weight-to-inner-skull-circumference ratio similar to the nonedema cases. Both equations are based on brain weight and CT-measured inner skull transverse and longitudinal distances.

This equation is represented by the red line in Fig. 3. We propose a conservative approach to evaluate brain edema cases by using this regression equation. Edema is likely when the measured brain weight is heavier than the calculated $y$ for the given head circumference measurements, and thus above the red line in Fig. 3.

Logistic regression was performed to rule out any confounding factors, including postmortem time, sex, body weight, height, and BMI (data not shown). There was a significant difference in age between the two groups ( $\mathrm{p}=0.031)$. However, this is thought to have been due to opportunistic 
sampling rather than actually affecting the development of edema. There was no correlation between age and brain-weight-to-inner-skull-circumference ratio (data not shown).

There was no significant intergroup difference in brain water content (Table 2, Fig. 4). A twosided Mann-Whitney $U$ test showed a significant difference in water content in the frontal lobes ( $\mathrm{p}=0.035)$. However, this was most likely due to one case in the edema group suffering severe head injuries with large frontal skull fractures and bleedings, since removing this case from the data set resulted in no significant difference between the groups (data not shown).

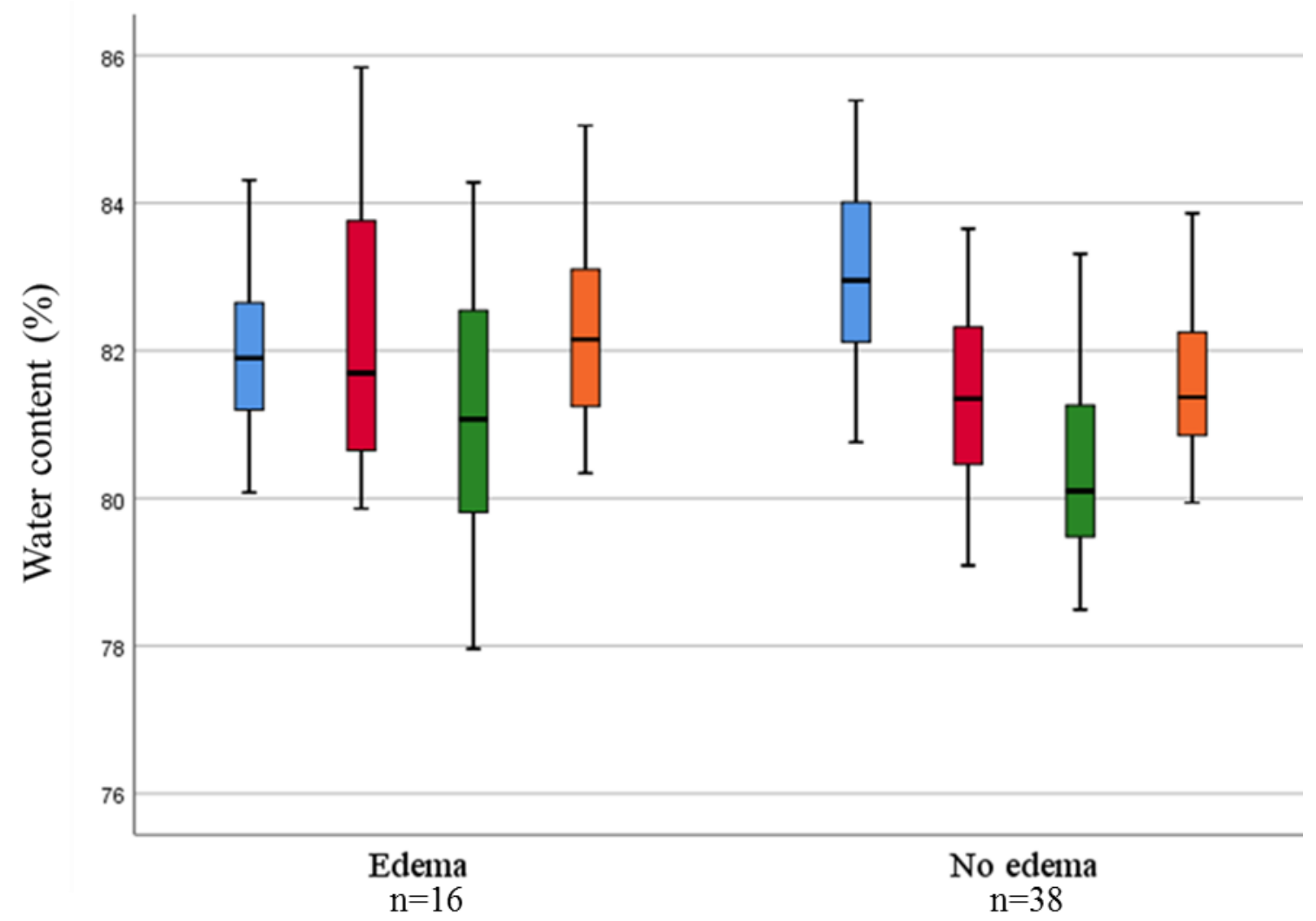

Fig. 4 Water content in the frontal lobes (blue), temporal lobes (red), occipital lobes (green), and the cerebellum (orange) in 16 cases with brain edema (including 4 late edema) and in 38 cases without edema. Each box contains the middle $50 \%$ of the values, the thick line indicates the median, and the bars indicate the maximum and minimum values. 


\section{Discussion}

The main finding of this study is the significant relationship between brain weight and inner skull circumference, and that the ratio between these parameters is significantly higher in cases with severe postmortem brain edema compared to cases with very little or no brain edema (Fig. 3). This indicates that a calculation based on the brain weight and the skull size can serve as a good marker for severe brain edema in postmortem diagnostics.

Weighing the brain is part of the basic autopsy procedure. The circumference of the interior of the skull is not commonly measured, but quite easily assessable. CT-scanning is now widely used as an adjunct to the forensic autopsy and stored images enable later measurements if not performed during the inner examination. In this study we found that brain weight differed significantly between subjects with and without brain edema (Table 2). However, the large variability in the weight of normal brains shows that brain weight alone is a poor indicator of postmortem brain edema in individual cases. Based on the findings in the present study, we propose an objective estimation of brain edema based on brain weight and of inner skull circumference. However, any estimation must be used with caution and in combination with clinical information and macroscopic and histological findings. An increased brain weight and brain-weight-to-inner-skull-circumference ratio may have other origins than edema. Agonal congestive brain swelling (increased blood volume in the brain), bleedings, or an increased amount of brain tissue (e.g., in brain tumors) may affect the brain weight and thus also the ratio. False-negative results could be due to loss of brain tissue due to pre-existing atrophy prior to the injury or due to a later stage of severe edema with widespread cell death. We observed that cases of fatal brain edema that had been on life-support for more than five days had lower brain-weight-to-inner-skull-circumference-ratio than expected. In these late edema cases the neuropathology also involved advanced markers of tissue death e.g. histiocytic reaction, reflecting loss of brain tissue, but the macroscopic appearance were that of severe edema with flattened gyri and filled sulci, compressed ventricles and/or signs of herniation. 
Radojevic et al. introduced a coefficient calculated as $\varepsilon=5.79 \times$ (longitudinal distance in the skull) $\times($ transverse distance in the skull)/brain weight (8). The constant 5.79 takes into account both the height of the skull as well as the density of brain tissue as determined based on cases included in their study. According to this method, a case with $\varepsilon<0.9484$ it could be diagnosed as cerebral edema with a reliability of $98.5 \%$. Their equation also uses interior skull measurements to approximate the skull volume. When we used this equation to calculate $\varepsilon$ for each of our cases (data not shown), the obtained values indicated incorrectly that all of them were brain edema. This discrepancy could be due to differences in the used measurements (Radojevic et al. used physical measurements of the actual skulls whereas our assessments were based on CT images), or to dissimilarities in skull anatomy between our population and theirs. Furthermore, Radojevic et al. diagnosed brain edema by evaluating hematoxylin-and-eosin-stained tissue sections. We consider that assessing brain edema based on a microscopic evaluation involves even more uncertainties than the scoring system applied in the present study.

Postmortem grading of brain edema based on macroscopic signs alone has been found to be problematic. Several authors have concluded that both macroscopic and morphological signs of brain swelling should be interpreted with caution $(6,7,11)$. In our diagnostic routine we evaluate edema by examining the brain immediately after removing it from the skull combined with evaluating the size of cerebral ventricles by CT. In many cases we see very clear signs of impression marks, which should indicate severe edema, but without any signs of compressed ventricles in the CT images. In other cases the ventricles are almost completely obliterated, including the anterior horns, while the brain surface looks normal in a visual examination. It is well known that a certain degree of brain swelling can occur as an agonal postmortem phenomenon due to blood congestion (3). The findings of the present study question whether some of the cases evaluated as edema were actually due to the postmortem redistribution of fluid within the brain tissue. Although we could not detect any consistency between water content and postmortem time, there are other reports of a significant increase in water content during the first 24 hours after death (12), which was attributed to liquefaction resulting from the breakdown of cerebral tissue. 
We found no significant differences in the gray-matter-to-white-matter radiodensity ratio between the groups (Table 2). This is consistent with previous findings (9), although the median ratio (and also the mean ratio, data not shown) was somewhat lower in our material (1.1 in both the edema and nonedema groups) than the mean ratios in the study of Berger et al. (1.4 in both groups) (9). When measuring the radiodensity in $\mathrm{HU}$ we experienced difficulties distinguishing between gray matter and white matter due to variations in image quality and postmortem changes. Evaluating the temporal horns on postmortem images was similarly difficult in our experience as nonradiologists, and was therefore not performed. It would appear that the difficulties of performing consistent evaluations of edema based on postmortem CT are similar that those based on visual examinations of the brain.

We found that the brain water content did not differ between the subjects with and without postmortem brain edema. This was a surprising finding that may indicate that taking eight samples from the brain surface was not sufficient for determining the total water content of the brain, which could have been due to the water distribution differing in the various parts and layers of the brain.

The samples used for determining the water content were collected from the outer surface of the brain, and so contained tissue from the cortical ribbon as well as some of the underlying whitematter tissue. Several authors have claimed that edema fluid is mainly found in the white matter $(6,13$, 14). Fully investigating the water content of the brain would require samples to be taken from deeper inside the brain. However, this approach would compromise the possibility of subsequent examinations of the fixed brain, and is therefore not feasible at present.

One limitation of the present study is the relatively low number of cases. It makes the statistical significance of our findings rather rough, and there is a need for additional cases in order to fully develop a model to distinguish mild brain edema from non-edema. A second limitation is that fixation and thorough neuropathological examination of the brain was only performed in cases macroscopically considered to be clear-cut edema. Thus, hidden pathology may have been missed in some of these cases. Some of our edema cases had traumatic brain injury. While local edema may develop around the lesions and injuries, the diffuse brain swelling may be related to vascular 
congestion rather than true edema. Sampling was carefully performed away from any lesions or damaged areas so as to avoid them interfering with the diagnostic examinations. This approach makes it possible that we missed the edematous areas of the brain or that these cases had been misclassified as global edema instead of diffuse brain swelling due to vascular congestion. All these cases were evaluated as edema both by premortem neuroradiology and by neuropathological examination with microscopic evaluation.

The development of an objective and reliable method for evaluating postmortem brain edema might have implications for several lines of diagnostics and research. It appears that mathematical models based on imaging and physical measurements can offer a supplement in the evaluation of brain edema, which otherwise is rather dependent upon the skills and experience of the examining physician. We document a clear correlation between the findings of macroscopic evaluations and the brain-weight-toinner-skull-circumference ratios. The roles of radiodensity measurements and water content determination in postmortem evaluation of edema are uncertain.

\section{Ethics}

This research project was approved by the Norwegian Regional Committee for Medical and Health Research Ethics. The right to access confidential information from the autopsy journals was granted by the Regional Public Prosecution Office in Oslo.

All next of kin were given the opportunity to register in a nonresearch exclusion register, and were explicitly informed that they could withdraw their deceased from this research study at any time.

\section{Conflict of interest}

There were no potential conflicts of interest with respect to the research, authorship, and/or publication of this article.

\section{Funding}


This work was supported by the Norwegian ExtraFoundation for Health and Rehabilitation and the Norwegian SIDS and Stillbirth Society (grant number 2016/FO77039).

\section{Acknowledgements}

We thank the Letten Centre (Department for Molecular Neuroscience, University of Oslo) for lending us their vacuum oven and former colleague Anne Lindboe for developing the water content determination method.

\section{References}

1. Klatzo I. Presidental address. Neuropathological aspects of brain edema. Journal of neuropathology and experimental neurology. 1967;26(1):1-14.

2. Ayata C, Ropper AH. Ischaemic brain oedema. Journal of clinical neuroscience. 2002;9(2):113-24.

3. Whitwell HL. Brain swelling and oedema, raised intracranial pressure and the non- perfused brain. In: Whitwell HL (ed) Forensic Neuropathology. London: Hodder Arnold; 2005. p. 10712.

4. Saukko P, Knight, B. Head and Spinal Injuries. In: Saukko P, Knight, B (eds) Knight's Forensic Pathology. London: CRC Press; 2016.

5. Rothig W, Schaarschmidt W. Linear relationship between body length and brain weight. Gegenbaurs morphologisches Jahrbuch. 1977;123(2):208-13. [Article in German].

6. Madro R, Chagowski W. An attempt at objectivity of post mortem diagnostic of brain oedema. Forensic science international. 1987;35(2-3):125-9.

7. Hausmann R, Vogel C, Seidl S, Betz P. Value of morphological parameters for grading of brain swelling. International journal of legal medicine. 2006;120(4):219-25.

8. Radojevic N, Radnic B, Vucinic J, Cukic D, Lazovic R, Asanin B, et al. Mathematical model in post-mortem estimation of brain edema using morphometric parameters. Journal of Forensic and Legal Medicine. 2017;45:21-8. 
9. Berger N, Ampanozi G, Schweitzer W, Ross SG, Gascho D, Ruder TD, et al. Racking the brain: detection of cerebral edema on postmortem computed tomography compared with forensic autopsy. European journal of radiology. 2015;84(4):643-51.

10. Shirota G, Gonoi W, Ishida M, Okuma H, Shintani Y, Abe H, et al. Brain Swelling and Loss of Gray and White Matter Differentiation in Human Postmortem Cases by Computed Tomography. PLOS ONE. 2015;10(11):e0143848.

11. Rothig W. The so-called pressure zone of the cerebellum. Gegenbaurs morphologisches Jahrbuch. 1976;122(6):882-907. [Artivle in German].

12. Leonard A, Vink R, Byard RW. Brain Fluid Content Related to Body Position and Postmortem Interval - An Animal Model. Journal of forensic sciences. 2016;61(3):671-3.

13. Alexander L, Looney JM. Physicochemical properties of brain, especially in senile dementia and cerebral edema: Differential ratio of skull capacity to volume, specific weight, water content, water-binding capacity and ph of the brain. Archives of Neurology \& Psychiatry. 1938;40(5):877-902.

14. Scheinker IM. Cerebral swelling; histopathology, classification and clinical significance of brain edema. Journal of neurosurgery. 1947;4(3):255-75. 